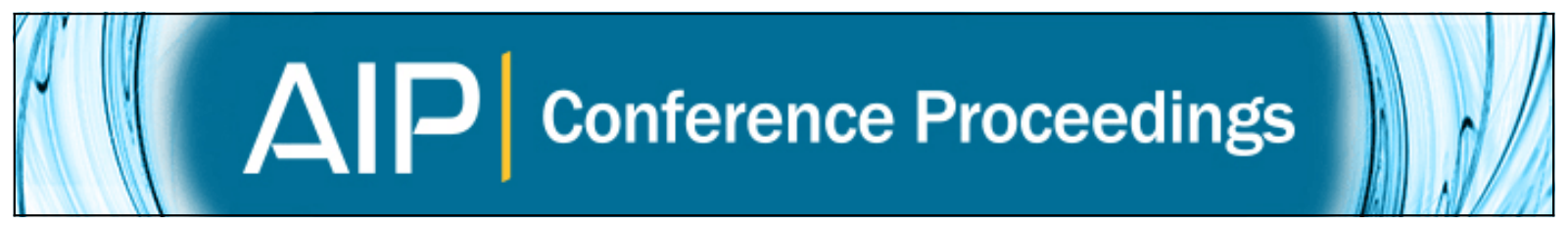

Proper Motions and Tangential Velocities for a Large Sample of Late-type M, L and T Dwarfs

Jacqueline K. Faherty, Adam J. Burgasser, Kelle L. Cruz, Michael M. Shara, Frederick M. Walter, and

Christopher R. Gelino

Citation: AIP Conference Proceedings 1094, 517 (2009); doi: 10.1063/1.3099162

View online: http://dx.doi.org/10.1063/1.3099162

View Table of Contents: http://scitation.aip.org/content/aip/proceeding/aipcp/1094?ver=pdfcov

Published by the AIP Publishing

Articles you may be interested in

Rotation and Activity in Late-type M Dwarfs

AIP Conf. Proc. 1094, 700 (2009); 10.1063/1.3099210

Decametric observations of active M-dwarfs

AIP Conf. Proc. 1094, 680 (2009); 10.1063/1.3099205

Clouds, Brightening and Multiplicity Across the $L$ Dwarf/T Dwarf Transition

AIP Conf. Proc. 1094, 501 (2009); 10.1063/1.3099158

Observation and modelling of dusty, low gravity $L$, and $M$ dwarfs

AIP Conf. Proc. 1094, 283 (2009); 10.1063/1.3099103

Large-scale magnetic topologies of $\mathrm{M}$ dwarfs

AIP Conf. Proc. 1094, 140 (2009); 10.1063/1.3099084 


\title{
Proper Motions and Tangential Velocities for a Large Sample of Late-type M, L and T Dwarfs
}

\author{
Jacqueline K. Faherty*,†, Adam J. Burgasser**, Kelle L. Cruz ${ }^{\ddagger}$, Michael M. \\ Shara $^{\dagger}$, Frederick M. Walter* and Christopher R. Gelino ${ }^{\S}$ \\ ${ }^{*}$ Stony Brook University Stony Brook, NY 11794-3800 \\ ${ }^{\dagger}$ American Museum of Natural History, Central Park West at 79th Street, New York, NY 10034 \\ ${ }^{* *}$ Massachusetts Institute of Technology, Cambridge, MA 02139 \\ $¥$ Astronomy Department, California Institute of Technology, Pasadena, CA 91125 \\ ${ }^{\S}$ Spitzer Science Center, California Institute of Technology, Pasadena, CA 91125
}

\begin{abstract}
We report proper motion measurements for 427 late-type M, L and T dwarfs, 332 of which have been measured for the first time. Combining these new proper motions with previously published measurements yields a sample of 841 M7-T8 dwarfs. We combined parallax measurements or calculated spectrophotometric distances and computed tangential velocities for the entire sample. We find that kinematics for the full and volume-limited $20 \mathrm{pc}$ samples are consistent with those expected for the Galactic thin disk, with no significant differences between late-type M, L, and $\mathrm{T}$ dwarfs. Applying an age-velocity relation we conclude that the average kinematic age of the 20 pc sample of ultracool dwarfs is older than recent kinematic estimates and more consistent with age results calculated with population synthesis models. We isolate subsets of the entire sample, including low surface-gravity dwarfs, unusually blue $\mathrm{L}$ dwarfs, and photometric outliers in $J-K_{s}$ color and investigate their kinematics. We find that the spectroscopically distinct class of unusually blue $\mathrm{L}$ dwarfs has kinematics clearly consistent with old age, implying that high surface-gravity and/or low metallicity may be relevant to their spectral properties. The low surface-gravity dwarfs are kinematically younger than the overall population, and the kinematics of the red and blue ultracool dwarfs suggest ages that are younger and older than the full sample, respectively.
\end{abstract}

Keywords: Astrometry- stars: low-mass- brown dwarfs- stars: fundamental parameters

PACS: 95.10.-a, 95.10.Jk,95.80.+p, 95.85.Jq, 97, 97.10.Wn, 97.20.Tr

\section{INTRODUCTION}

Kinematic analyses of stars have played a fundamental role in shaping our picture of the Galaxy and its evolution. One population that has yet to have its kinematics exploited is the recently discovered population of very low-mass ultracool dwarfs (UCDs). These objects, which include those that do not support stable hydrogen fusion (Kumar 1), occupy the late-type $M$ through $T$ dwarf spectral classifications [e.g., 2, and references therein]. Their very recent discovery has largely precluded astrometric measurements which require several-year baselines to produce useful measurements. We have initiated the Brown Dwarf Kinematics Project (BDKP) which aims to measure the positions and $3 \mathrm{D}$ velocities of all known $\mathrm{L}$ and T dwarfs within $20 \mathrm{pc}$ of the Sun and selected sources of scientific interest at larger distances (e.g. low surface-gravity dwarfs, subdwarfs). In this article we explore some of the results of our recent kinematic survey of ultracool dwarfs. 


\title{
SAMPLE AND ANALYSIS
}

\author{
Sample Selection
}

We re-imaged 427 late-type M, L and T dwarfs from March 2006 through April 2008 in order to obtain second epoch images and measure proper motions for a large sample of UCDs. As of June 2008 and including all of the measurements reported in this article, 570 of the $634 \mathrm{~L}$ and T dwarfs (primarily from the dwarfarchives compendium) and 277 of the 456 late-type M dwarfs (primarily from Cruz et al. 2003, 2007) in our sample have measured proper motions. For information on the astrometric calibration of images and proper motion measurements please see Faherty et al. 3 .

True space velocities are a more fundamental measure of an object's kinematics than apparent angular motions, so proper motions for the complete sample were converted to tangential velocities using astrometric or spectrophotometric distances. If a parallax measurement was unavailable, the distances for the M7-L4.5 dwarfs were calculated using the absolute 2MASS J magnitude/spectral type relation in Cruz et al. [4] and the distances for the L5 -T8 dwarfs were calculated using the absolute MKO K magnitude/spectral type relation in Burgasser [5]

\section{Full and 20pc Samples}

We have conducted our kinematic analysis on two samples: the full astrometric sample and the 20 pc sample. Based on the distribution of distances for UCDs we have chosen $20 \mathrm{pc}$ as the volume-limiting distance (see Faherty et al. 3) .

We find no difference between the two samples, with median $V_{\text {tan }}$ values of $26 \mathrm{~km} \mathrm{~s}^{-1}$ and $29 \mathrm{~km} \mathrm{~s}^{-1}$ and $\sigma_{\text {tan }}$ values of $23 \mathrm{~km} \mathrm{~s}^{-1}$ and $25 \mathrm{~km} \mathrm{~s}^{-1}$ for M7-T9 within the full sample and the $20 \mathrm{pc}$ sample respectively. Within spectral class bins, namely the M7-M9, L0-L9, or T0-T9 groupings, we find no significant kinematic differences. This indicates that we are sampling a single kinematic population regardless of distance and spectral type.

We conduct a crude $\mathrm{U}, \mathrm{V}, \mathrm{W}$ analysis on the sample by minimizing the importance of the radial velocity component and restricting the sample by an objects angular separation from a Galactic, $\mathrm{X}, \mathrm{Y}$, or $\mathrm{Z}$ axis and find that the velocity dispersions of the population fit what is expected for objects in the thin disk. We measure $\left(\sigma_{U}, \sigma_{V}, \sigma_{W}\right)=(28,22,17)$ $\mathrm{km} \mathrm{s}^{-1}$ ).

\footnotetext{
1 The coefficients of this polynomial relation reported in Burgasser [5] did not list sufficient significant digits, yielding a slightly different numerical relation than that used in the paper's analysis. The coefficients as defined should be $\left\{c_{i}\right\}=[10.4458,0.232154,0.0512942,-0.0402365,0.0141398,-0.00227108$, $0.000180674,-6.98501 \mathrm{e}-06,1.05119 \mathrm{e}-07]$, where $M_{K}=\sum_{i=0}^{6} c_{i} \mathrm{SpT}^{i}$ and SpT(T0) $=10, \operatorname{SpT}(\mathrm{T} 5)=15$, etc.
} 


\section{Red and Blue Photometric Outliers}

The large number of late-type $\mathrm{M}, \mathrm{L}$, and $\mathrm{T}$ dwarfs discovered to date has revealed a broad diversity of colors and spectral characteristics, including specific subgroups of peculiar sources that are likely related by their common physical properties. To investigate our sample for kinematically distinct photometric outliers, we looked for objects that deviated significantly in $\mathrm{J}-\mathrm{K}_{S}$ color space for their spectral bin (See Faherty et al. 3 for details).

Amongst the full sample, we found 16 blue photometric outliers and 29 red photometric outliers. The blue outliers have a median $V_{\tan }$ value of $53 \mathrm{~km} \mathrm{~s}^{-1}$ and a $\sigma_{\tan }$ of $47 \mathrm{~km} \mathrm{~s}^{-1}$ while the red outliers have a median $V_{\text {tan }}$ value of $26 \mathrm{~km} \mathrm{~s}^{-1}$ and a $\sigma_{\text {tan }}$ of $16 \mathrm{~km} \mathrm{~s}^{-1}$. Figure 1 shows the tangential velocity vs. J- $K_{s}$ deviation for all objects in the sample with the dispersions of the red and blue outliers highlighted. There is a clear trend for $V_{\tan }$ values to decrease from objects that are blue for their spectral type to those that are red.

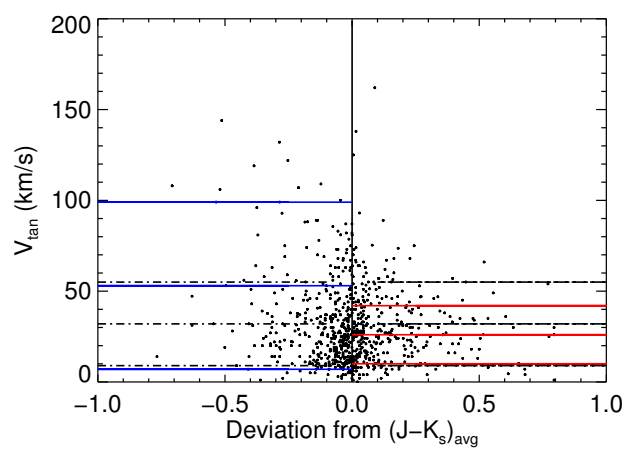

FIGURE 1. A scatter plot showing $V_{t a n}$ as a function of the deviation in $J-K_{s}$ color from the average at a given spectral type.

\section{Low Surface - gravity and Unusually Blue L Dwarfs}

A number of ultracool dwarfs that exhibit low surface-gravity features have been reported in the literature within the past few years. We examine the kinematics of 37 low surface-gravity dwarfs in this paper and find the median $V_{\text {tan }}$ value for this subgroup is $18 \mathrm{~km} \mathrm{~s}^{-1}$ and the $\sigma_{\text {tan }}$ value is $15 \mathrm{~km} \mathrm{~s}^{-1}$, making them a kinematically distinct population from either the full or $20 \mathrm{pc}$ sample.

A subgroup of unusually blue L dwarfs (UBLs) has been distinguished based on strong near-IR $\mathrm{H}_{2} \mathrm{O}$ and $\mathrm{FeH}$ spectral features but otherwise normal optical spectra. Burgasser et al. [6] identify ten objects that comprise this subgroup (see Table 6 in B08). The median $V_{\text {tan }}$ value for this subgroup is $99 \mathrm{~km} \mathrm{~s}^{-1}$ with $\sigma_{\text {tan }}$ of $47 \mathrm{~km} \mathrm{~s}^{-1}$ and the UBLs consist of dwarfs with the largest $V_{t a n}$ values measured in this kinematic study. These kinematic results strengthen the case that the UBLs represent an older 
population and that the blue near-IR colors and spectroscopic properties of these objects are influenced by large surface-gravity and/or slightly subsolar metallicities.

\section{ON THE AGES OF THE ULTRACOOL DWARF POPULATIONS}

While individual $V_{\text {tan }}$ measurements cannot provide individual age determinations due to scatter and projection effects, the random motions of a population of disk stars are known to increase with age. This effect is known as the disk age-velocity relation (AVR). We conducted a direct kinematic comparison with nearby stellar populations to draw conclusions about the kinematic distinguishability of our ultracool dwarf sample and used the AVR to infer information about an age comparison. We compared the kinematics of a $20 \mathrm{pc}$ sample of F,G,K, and early M stars from Soubiran et al. [7], Kharchenko et al. [8] and Nordström et al. [9] using a limiting proper motion of 25 mas/yr to our $20 \mathrm{pc}$ sample and examined the resultant median $V_{t a n}, \sigma_{t a n}$, and $V_{t o t}, \sigma_{t o t}$ values (where $V_{\text {tot }}$ comes from the $\mathrm{U}, \mathrm{V}, \mathrm{W}$ velocities). The velocity dispersions are in good agreement between the UCDs and nearby stellar populations. With AVR ages of 3-8 Gyr calculated from dispersions, we conclude that our 20 pc sample is kinematically indistinct from other nearby stellar populations and hence is in good agreement with ages predicted in population synthesis models and not kinematically younger.

We find that the kinematics for the red outliers as well as the low-gravity dwarfs are consistent with a younger population of ultracool dwarfs whereas the kinematics for the blue outliers and UBLs are consistent with an older population. The $\sim 38$ Gyr mean age for the blue outliers is misleading. It indicates a large divergence from the full and $20 \mathrm{pc}$ samples but also indicates that the AVR must be incorrect for these objects. The more informative number in this case is the median $V_{\text {tan }}$ which, at $56 \mathrm{~km} / \mathrm{s}$, is nearly twice the expected value for the thin disk. The blue photometric outliers most likely belong to an older population of the Galaxy such as the thick disk or the halo. The Wielen AVR is only valid for thin disk objects and we are unaware of an equivalent age relation for the halo or thick disk population. We suggest that kinematic studies of UCDs to date that have reported ages on the order of $\sim 1$ Gyr, may have been plagued by small number statistics or a bias in the sample analyzed.

\section{REFERENCES}

1. S. S. Kumar, $A J$ 67, 579-+ (1962).

2. J. D. Kirkpatrick, $A R A \& A$ 43, 195-245 (2005).

3. J. K. Faherty, A. J. Burgasser, K. L. Cruz, M. M. Shara, F. M. Walter, and C. R. Gelino, ArXiv e-prints 809 (2008), 0809.3008

4. K. L. Cruz, I. N. Reid, J. Liebert, J. D. Kirkpatrick, and P. J. Lowrance, AJ 126, 2421-2448 (2003).

5. A. J. Burgasser, ApJ 659, 655-674 (2007), arXiv:astro-ph/0611505

6. A. J. Burgasser, D. L. Looper, J. D. Kirkpatrick, K. L. Cruz, and B. J. Swift, ApJ 674, 451-465 (2008), [B08], arXiv:0710.1123

7. C. Soubiran, O. Bienaymé, and A. Siebert, A\&A 398, 141-151 (2003).

8. N. V. Kharchenko, A. E. Piskunov, and R.-D. Scholz, VizieR Online Data Catalog 3239, 0-+ (2004).

9. B. Nordström, M. Mayor, J. Andersen, J. Holmberg, F. Pont, B. R. Jørgensen, E. H. Olsen, S. Udry, and N. Mowlavi, A\&A 418, 989-1019 (2004), arXiv:astro-ph/0405198. 